\title{
Mindfulness y educación física en la población universitaria. Una revisión sistemática \\ Mindfulness and physical education in University population. A systematic review
}

\author{
*Irene López Secanell, **Javier Gené Morales, **Nerea Hernaiz Agreda \\ **Florida Universitària (España), **Universidad de Valencia (España)
}

\begin{abstract}
Resumen. El objetivo de este estudio fue revisar las conclusiones de los artículos publicados en los últimos seis años sobre el uso del mindfulness en la población universitaria. Además, se examinaron cuántos de estos estudios se aplican dentro del campo de la Educación Física Universitaria. Se analizaron los artículos publicados desde 2014 hasta 2020 mediante las bases de datos ERIC, Taylor and Francis, Web of Science y SCOPUS, en base a los requerimientos de PRISMA y el modelo PICO. Se extrajo la información que sigue de los artículos seleccionados: autor, año, país, idioma, sujetos, duración, tipo de investigación (cualitativa/cuantitativa), programa de mindfulness utilizado, propósito y resultados. Como resultado se incluyeron 11 artículos. Ningún estudio fue publicado en España ni en español, siendo todos publicados en inglés y la mayoría (6 artículos) en el Reino Unido. La duración de las intervenciones varía entre 2 y doce semanas. De los 11 artículos, nueve fueron de diseño cuantitativo, uno cualitativo y uno mixto. Los resultados confirman los beneficios del mindfulness en los estudiantes universitarios mejorando su salud psicológica (estrés, ansiedad, depresión, motivación, autoaceptación) y también a nivel cognitivo y emocional. Los programas de mindfulness utilizados fueron MBI, MBCT, MBSR, MBSH y MTP. Por tanto, a pesar de los beneficios corroborados del mindfulness con la población universitaria, son escasos los estudios en el área de la Educación Física Universitaria y se estima pertinente la realización de intervenciones de mindfulness en universidades españolas para certificar su validez en estudiantes con dichas características socioculturales.
\end{abstract}

Palabras clave: mindfulness, psicología, deporte, actividad fisca, educación física, estudiantes universitarios.

\begin{abstract}
The aim of this study was to review the conclusions of published papers in the last six years on the use of mindfulness in the university population. In addition, how many of these studies are applied within the field of University Physical Education were examined. Articles published from 2014 to 2020 were analysed using the ERIC, Taylor and Francis, Web of Science and SCOPUS databases, based on the PRISMA requirements and the PICO model.The following information was extracted from the selected articles: author, year, country, language, individuals, duration, type of research (qualitative / quantitative), mindfulness program used, purpose and results. As a result, 11 articles were included. Neither study was published in Spain or in Spanish, all of which were published in English and the majority (6 articles) in the United Kingdom. The duration of the interventions varies between 2 and 12 weeks. Of the 11 articles, nine were of quantitative design, one qualitative and one mixed. The results confirm the benefits of mindfulness in university students improving their psychological health (stress, anxiety, depression, motivation, self-acceptance) and also on a cognitive and emotional level. The mindfulness programs used were MBI, MBCT, MBSR, MBSH and MTP.Therefore, despite the corroborated benefits of mindfulness with the university population, there are few studies in the area of University Physical Education and it is considered pertinent to carry out mindfulness interventions in Spanish universities to certify its validity in students with these sociocultural features. Keywords: mindfulness, psychology, sport, physical activity, physical education, university students.
\end{abstract}

\section{Introducción}

Según Solé, Carrança, Serpa y Palmi (2014), el mindfulness debe ser una herramienta que se siga extendiendo en el ámbito deportivo, dado que, en otras disciplinas como la psicología, queda demostrada su competencia. Las investigaciones realizadas evidencian cómo el uso del mindfulness en el deporte favorece una me-

Fecha recepción: 08-12-20. Fecha de aceptación: 31-05-21

Irene López Secanell

irlopez@florida-uni.es jora del rendimiento del deportista (Palmi \& Solé, 2016; Gooding \& Gardner, 2009). Además, se han constatado mejoras en las expectativas de los deportistas hacia la competición (Kauffman, Glass \& Arnkoff, 2009; Palmi \& Solé, 2016; Worthen \& Luiselli, 2016), así como mejoras en su relajación (Dunn, Hartigan \& Mikulas, 1999; Kauffman, et al., 2009), atención y consciencia durante el juego (Worthen \& Luiselli, 2016), variables psicológicas como la ansiedad (Kauffman, et al., 2009; Palmi \& Solé, 2016) y también una reducción del burnout en deportistas (Garcés, De Francisco \& Arce, 2012). Además, se ha encontrado que el mindfulness mejora a tra- 
vés de la compasión consciente y las intervenciones basadas en la aceptación, como el programa de fútbol basado en la atención plena Mindfulness-Based Soccer Program (MBSoccerP) que reduce muchos de los síntomas asociados a la ansiedad y ayuda a disminuir pensamientos negativos o hacer que desaparezcan de nuestros pensamientos (Carraça et al., 2018, 2019).

Cuando una persona está atenta y es consciente de las experiencias de sí misma en el momento presente con aceptación y apertura, refleja el estado de atención plena. Existen asociaciones positivas entre un estado de atención plena y un estado de motivación autónoma para las actividades cotidianas, como la actividad física. Cuando se está intrínsecamente motivado, se elige estar activo por la forma positiva en que la actividad física nos hace sentir o por las recompensas intrínsecas que se obtienen al participar. El disfrute y la satisfacción derivados de las sensaciones físicas de la actividad física o el sentimiento psicológico de logro durante la actividad física representan estados internos que pueden ser características inherentemente gratificantes (Ryan \& Deci, 2007).

Los resultados positivos que ha demostrado tener el mindfulness en la actividad física, han originado que en la actualidad esta técnica sea utilizada también en Educación Superior para que los estudiantes universitarios puedan beneficiarse de sus resultados a nivel psicológico, emocional y cognitivo.

\section{Aproximación al concepto de mindfulness}

La atención plena se basa en «prestar atención de una manera particular: intencionalmente, en el momento presente y sin juzgar» (Kabat-Zinn, 1994, p.4), en lugar de desafiar pensamientos y emociones negativas, como en las terapias cognitivo-conductuales. Una característica de esta definición es el reconocimiento de un modo de práctica secular e instrumental, mediante el cual se pide a quienes participan en la atención plena que reconozcan y acepten las emociones presentes, dedicando tiempo a aprender a observar los pensamientos como abstracciones sin causar angustia emocional (KabatZinn, 2003).

Se reconocen tres componentes dentro del mindfulness que ocurren de manera simultánea: intención, atención y actitud (Shapiro, Carlson, Astin \& Freedman, 2006). La intención está relacionada con los resultados y hace que las personas recuerden por qué están practicando la atención plena. La atención identifica los diferentes ambientes de los cuales esas personas forman parte y las experiencias internas de momento a momento. Mientras que la actitud es necesaria para incluir las cualidades internas personales en la atención (Kabat-Zinn, 2003; Shapiro \& Carlson, 2017).

Siguiendo con los componentes que definen la atención plena, nos centraremos en el concepto del mindfulness en relación a la autoconexión y el bienestar. Por un lado, la atención plena es un predictor fuerte y consistente del bienestar. Las personas que practican el mindfulness informan de varios efectos positivos y duraderos en el tiempo, como percepciones sobre agotamiento laboral (Fortney, Luchterhand \& Zakletskaia, 2013) y calidad del sueño (Hülsheger, et al., 2014). Otros indicadores que también se han reportado con relación a la práctica de la atención plena son una mayor sensación de bienestar y compasión (Baer, Lykins \& Peters, 2012), una mayor calidad de vida, una disminución de la depresión, la ansiedad, el dolor crónico y la discapacidad física, y mejores patrones de afrontamiento (Grossman, Niemann, Schmidt \& Walach, 2004).

\section{Beneficios del mindfulness en la actividad físi- ca y el deporte en población adulta}

Desde la disciplina de la psicología el mindfulness ha probado sus numerosos beneficios ya comentados. Por este motivo en otras áreas como la del deporte se ha implementado la técnica de la atención plena con distintas intervenciones y programas. Se destaca el Mindful Sport Performance Enhacement (MSPE) de Kauffman et al. (2009), puesto que mejora el rendimiento deportivo a través de la conciencia plena. Éste se focaliza en enseñar la aplicación del mindfulness en el deporte mediante la práctica repetida y la meditación adaptada a cada juego. El programa se puso en práctica en el estudio de Minkler, Glass, y Hut (2020) y se determinó que había mejoras significativas en la consciencia, la ansiedad, la satisfacción, el rendimiento deportivo y las dificultades en la regulación de las emocionales. En otras investigaciones el papel de la conciencia plena también ha recibido atención por su potencial para promover la actividad física en relación al bienestar de la persona y otros elementos (Schneider, Malinowski, Watson \& Lattimore, 2019). Además, en el estudio de Trujillo-Torrealva y Reyes-Bossio (2019) deportistas adultos disminuyeron sus niveles de ansiedad somática y cognitiva y aumentaron su autoconfianza antes de las competiciones a través del programa de intervención Mindfulness For Athlete Wellbeing (MFAW). Sin embargo, la investigación sobre la atención plena y la actividad física no es muy extensa y se ha de ahondar más en los beneficios que esta técnica tiene sobre el deporte. 
La atención plena puede desempeñar un papel en la promoción o el mantenimiento de la actividad física porque apoya tanto la atención como la aceptación. Estas características cognitivas pueden tener la capacidad de anular o inhibir tendencias habituales no deseadas y así mejorar el autocontrol (Whitmarsh, Uddén, Barendregt, \& Petersson, 2013). Según Hart, Ivtzan y Hart (2013), los estudios sobre atención plena suelen incluir capacitación en la regulación de la atención y la adopción de una orientación abierta y de aceptación. El ejercicio de atención plena contribuye a la autorregulación efectiva de la actividad física (Bandura, 2005).

En un contexto de rendimiento deportivo, la atención plena puede no tener un efecto directo, pero sí puede ayudar a través de variables indirectas al tratarse de un concepto complejo y multicomponente (Birrer, Röthlin, \& Morgan, 2012; Gardner, 2009). Basándose en la investigación contemporánea sobre mindfulness, Birrer et al. (2012) estudiaron cómo los aspectos de la práctica pueden conducir a nueve mecanismos de impacto positivo específicos en el deporte (atención directa, actitud, aclaración de valores, autorregulación y regulación de emociones negativas, claridad, exposición, flexibilidad, desapego y menos rumia). En esta investigación se conectaron estos mecanismos con once dominios de habilidades psicológicas que pueden favorecer el rendimiento atlético (desarrollo personal y habilidades para la vida, habilidades personales, recuperación, afrontamiento, motivación, manejo del dolor, de atención, regulación de la excitación, habilidades perceptivocognitivas, control motor y habilidades de comunicación y liderazgo). Por lo que se indicó que unas habilidades influían positivamente en otras, activando los mecanismos que ayudan en el rendimiento deportivo.

\section{El mindfulness en población universitaria}

Aunque la investigación ha demostrado los beneficios de la atención plena para el bienestar de los estudiantes, muy pocos estudios han examinado la base fenomenológica de cómo los estudiantes universitarios experimentan y llegan a esta conciencia de la realidad. La principal justificación de este incremento de estudios radica en los beneficios de utilizar el mindfulness para mejorar la salud mental (física, social, emocional y cognitiva) de los estudiantes universitarios (Ergas \& Hadar, 2019). Este incremento de la investigación en torno al mindfulness ha permitido evidenciar algunos de sus beneficios a nivel psicológico, emocional y cognitivo en este colectivo, como por ejemplo la re- ducción de los niveles de estrés (Anand \& Sharma, 2014).

Kaufman (2017), basándose en la teoría de la educación transformadora, sostiene que la integración del mindfulness en la educación superior puede promover la interdependencia y el privilegio, aumentando así la conciencia crítica. Por otro lado, en el estudio de Mahalingam y Rabelo (2019) se practicó mindfulness con estudiantes universitarios dos veces por semana durante un período de siete semanas y obtuvieron resultados relacionados con el progreso en la creatividad, la autocompasión, la compasión hacia los demás, el bienestar psicológico y la regulación emocional. Por lo que se determinó que la integración de las prácticas de atención plena en la educación superior es fundamental para el desarrollo integral del aprendizaje y el bienestar de los estudiantes. Las prácticas de atención plena ayudarán a los estudiantes a enfrentar los factores estresantes asociados de manera única con la edad adulta emergente, como la comparación social y la desconexión. En líneas similares, en Aránega, Sánchez y Pérez (2019) se concluyó que los participantes dormían mejor y tenían mayores niveles de energía para afrontar el día. Además, la mayoría había dejado de sufrir trastornos alimentarios. Los participantes también mostraron una mejor capacidad para enfrentar problemas y responsabilidades, superando obstáculos y excusas que les impedían hacer todo lo posible para alcanzar los objetivos que se habían marcado antes de empezar con las sesiones de mindfulness. Asimismo, la práctica de aikido con estudiantes universitarios de Educación Física en la investigación de Cuéllar et al. (2018) constató la mejora de su atención plena y su relación directa con esta variable, así como la disminución de la ansiedad de los participantes en el programa.

En el estudio de Mrazek, Franklin, Phillips, Baird y Schooler (2013) se encontró el beneficio de la atención plena, debido a que los estudiantes universitarios de la investigación redujeron la distracción mental y mejoraron la capacidad de su memoria de trabajo consiguiendo así un mejor rendimiento académico. La investigación de Greeson, Juberg, Maytan, James y Rogers (2014) demostró también que practicar mindfulness y meditación dentro del programa académico reportaba numerosos beneficios, como la reducción del estrés.

Finalmente, se han podido encontrar algunos estudios realizados con muestra compuesta por universitarios españoles en los que se pone en relación la atención plena y la educación física. Todos ellos aluden a la necesidad y pertinencia de seguir estudiando los efectos de 
estas dos variables con programas que puedan aplicarse a estudiantes universitarios dentro del contexto español. En Gallego et al. (2014) se evidenció que los ejercicios de mindfulness y algunos ejercicios individuales de relajación de educación física podrían ayudar a reducir las manifestaciones de estrés y ansiedad causadas por los exámenes en los estudiantes de la Universidad de Almería. También en Hernández-Amorós y Urrea-Solano (2019) se investigaron los efectos de un taller de mindfulness con ejercicios físicos de relajación en estudiantes de la Universidad de Alicante que reportaron resultados positivos en los estudiantes tales como la adquisición de destrezas y habilidades para gestionar las emociones, mayor relajación y bienestar psicológico y reducción del estrés y la ansiedad, fomentando de este modo el desarrollo personal y académico de los participantes. En una investigación reciente con diferentes estudiantes de universidades valencianas (MartínezRubio et al., 2020) se concluyó que la práctica de mindfulness en combinación con ejercicios físicos puede ayudar a reducir el agotamiento en estudiantes universitarios.

Aunque la relación entre la actividad física, deporte y mindfulness es evidente, nuestra hipótesis está centrada en los escasos estudios realizados en España, donde se utiliza esta técnica en población adulta y, específicamente, en universitarios. Por este motivo, este trabajo plantea dos objetivos. El primero se centra en llevar a cabo una revisión sistemática de los artículos publicados desde el año 2014 al 2020 sobre el uso del mindfulness en los estudiantes universitarios. El segundo versa en saber cuántas de las investigaciones examinadas se dedican a aplicar el mindfulness en la disciplina de la Educación Física y, concretamente, en el ámbito universitario.

\section{Material y método}

Para llevar a cabo el abordaje del estudio nos hemos basado en el diseño de investigación de Barba-Martín, Bores-García, Hortigüela-Alcalá, y González-Calvo (2020) donde se realiza una revisión sistemática utilizando la guía PRISMA (Urrútia \& Bonfill, 2010) y la estrategia PICO (Participants, Intervention, Comparations, Outcomes).

\section{Fuentes de búsqueda}

Las bases de datos utilizadas para la pesquisa fueron: ERIC, Taylor and Francis, Web of Science y SCOPUS. Los descriptores incluidos en las búsquedas fueron «aten- ción plena», «mindfulness»y «educación física», relacionándose entre sí a través del operador booleano «OR». También se utilizó el operador «AND» combinado con términos como «universidad»y «estudiantes universitarios», para afinar la búsqueda con respecto a la población objetivo. Se consideró incorporar el descriptor «atención plena» junto con «mindfulness», ya que la revisión teórica previa realizada nos mostró que en el idioma español se consideran conceptos sinónimos.

\section{Criterios de inclusión-exclusión}

En esta revisión para la selección de los estudios se especificaron, junto con las bases de datos y descriptores, los siguientes criterios de inclusión: a) Artículos publicados en revistas indexadas de JCR o SJR; b) Artículos en inglés o español; c) Artículos en los que se implementa el mindfulness en población universitaria; d) Artículos en los que se utiliza el mindfulness en el ámbito de la Educación Física.

Para el análisis bibliográfico definitivo se excluyeron todos los elementos duplicados y aquellos artículos cuya intervención de mindfulness no se aplicaba en una muestra de estudiantes universitarios. Tampoco se incluyeron en el análisis los artículos de revisión.

\section{Procedimiento y selección de estudios}

La investigación se inició en febrero de 2020, tras una búsqueda inicial teórica para delimitar el problema de investigación y los objetivos, con el diseño de los criterios de selección de artículos, así como la elección de las bases de datos a utilizar. Después de formular las estrategias de búsqueda, se procedió a la pesquisa inicial de artículos. La búsqueda de artículos se dio por concluida el mes de julio de 2020. Con la especificación de los criterios y las bases de datos se inició el examen de los artículos obtenidos, encontrándose un total de 224

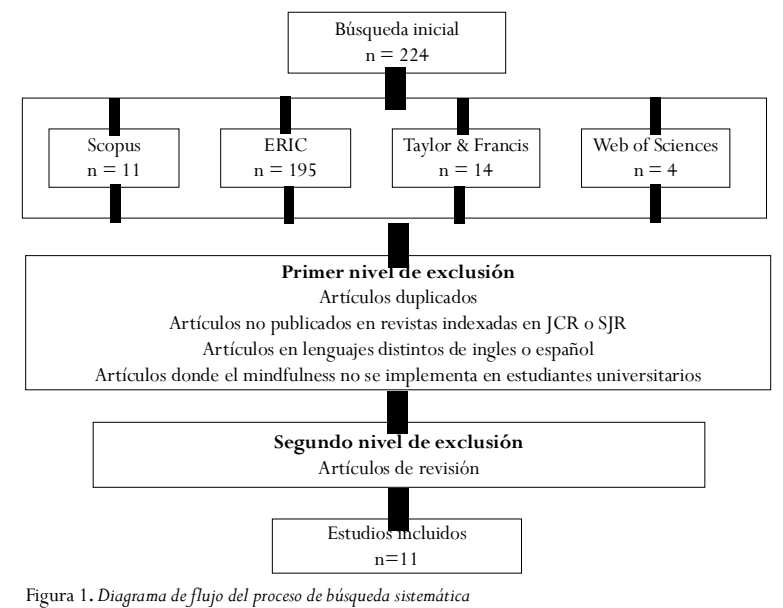

Retos, número 42, 2021 ( $4^{\circ}$ trimestre) 
publicaciones que correspondían a los descriptores antes mencionados (ver figura 1). Tras aplicar los criterios de inclusión-exclusión citados anteriormente, se seleccionaron 19 artículos. Finalmente, se descartaron los artículos con metodología de revisión bibliográfica y se seleccionaron de manera definitiva los 11 artículos que se incluyen en el análisis.

\section{Calidad de los estudios}

Para analizar la calidad de los 11 artículos seleccionados, se utilizaron los criterios incluidos en la tabla 1 (grado de inclusión en JCR o SJR; detalle del proceso metodológico utilizado; número de participantes; duración del estudio; y nivel de calidad de JCR). Cada uno de los investigadores rellenó la tabla de manera autónoma para evitar sesgos y posteriormente se discutió y acordó la puntuación final de los artículos.

\section{Resultados}

En primer lugar, se muestra la tabla 1 con el análisis de calidad de cada uno de los 11 estudios. Se observó que las puntuaciones de todos los artículos se encontraban entre cinco y nueve.

En este punto, se diseñó la tabla 2 con las características y datos más relevantes de los artículos seleccionados para la investigación (Autor y año, país e idioma, sujetos y duración del estudio, tipo de investigación [cualitativa/cuantitativa], programa de mindfulness aplicado, propósito y resultados).

Cabe destacar que ningún estudio fue publicado en España ni en español, siendo todos publicados en inglés y la mayoría (seis artículos) en el Reino Unido. El tamaño muestral de las investigaciones varía entre 22 y 284 sujetos, y la duración de las intervenciones entre dos y 12 semanas (tres estudios fueron transversales). De los 11 artículos, nueve fueron de diseño cuantitativo, uno cualitativo y uno mixto. Los programas de aten-

Tabla 1

\begin{tabular}{|c|c|c|c|c|c|c|}
\hline Autor (año) & $\mathrm{JCR} / \mathrm{SJR}$ & Metodología & $\begin{array}{c}\text { Duración } \\
\text { intervención }\end{array}$ & $\begin{array}{c}\text { Muestra } \\
\text { total }\end{array}$ & $\begin{array}{c}\text { Nivel de } \\
\text { calidad }\end{array}$ & Total \\
\hline $\begin{array}{ll}1 & \text { Stevenson, Millings, \& Emerson (2019) } \\
\end{array}$ & 1,2 & 2 & Estudio transversal & 2 & SJR/ JCR & 7 \\
\hline 2 Cavanagh et al. (2018) & 1,2 & 2 & 2 & 2 & $\mathrm{SJR} / \mathrm{JCR}$ & 9 \\
\hline 3 Gu, Cavanagh, \& Strauss (2018) & 1,2 & 2 & 2 & 2 & $\mathrm{SJR} / \mathrm{JCR}$ & 9 \\
\hline 4 James \& Rimes (2018) & 1,2 & 2 & 0 & 2 & $\mathrm{SJR} / \mathrm{JCR}$ & 7 \\
\hline 5 Brzozowski, Gillespie, Dizon, \& Mitchell (2018) & 1,2 & 2 & Estudio transversal & 2 & $\mathrm{SJR} / \mathrm{JCR}$ & 7 \\
\hline 6 Solhaug et al. (2016) & 1,2 & 2 & 2 & 1 & SJR/ JCR & 8 \\
\hline 7 Senker, Fries, \& Grund (2020) & 1,2 & 2 & 0 & 2 & $\mathrm{SJR} / \mathrm{JCR}$ & 7 \\
\hline $8 \quad$ Wimmer, Bellingrath, \& von Stockhausen (2020) & 1,2 & 2 & 0 & 2 & $\mathrm{SJR} / \mathrm{JCR}$ & 7 \\
\hline 9 Moore et al. (2020) & 1,2 & 2 & 1 & 1 & SJR/ JCR & 7 \\
\hline 10 Alzahrani et al. (2020) & 1,2 & 1 & Estudio transversal & 2 & SJR/ JCR & 6 \\
\hline 11 Turner et al. (2020) & 1,2 & 1 & 0 & 2 & $\mathrm{SJR} / \mathrm{JCR}$ & 6 \\
\hline \multicolumn{7}{|c|}{$\begin{array}{l}\text { Notas. Índice de evaluación basado en Barba-Martín et al. (2020). JCR/SJR (ffue el estudio publicado en revista indexada en } \\
\text { JCR o SJR?): 0: no indexado, 1: indexado en SJR y 2: indexado en JCR; Metodología (eel artículo informó en detalle sobre el } \\
\text { proceso metodológico utilizado?): 0: no informado, 1: informado pero impreciso (no completamente), y 2: descripción } \\
\text { exhaustiva informada; Duración: 0: menor que ocho sesiones, 1: de nueve a } 14 \text { sesiones, y 2: más de } 15 \text { sesiones; Muestra total } \\
\text { (número de participantes): 0: menos de } 10 \text { participantes, 1: de } 10 \text { a } 50 \text { participantes, y 2: más de } 50 \text { participantes; Nivel de } \\
\text { calidad: JCR: Informe de Citaciones de Revistas; SJR: Ranking de Revistas Scimago. }\end{array}$} \\
\hline
\end{tabular}

ción plena utilizados fueron intervenciones basadas en mindfulness (MBI; del inglés mindfulness-based interventions), terapia cognitiva basada en mindfulness (MBCT; del inglés mindfulness-based cognitive therapy), reducción del estrés a través del mindfulness (MBSR; del inglés mindfulness-based stress reduction), autoayuda basada en mindfulness (MBSH; del inglés mindfulnessbased self help), programa de entrenamiento en mindfulness (MTP; del inglés mindfulness training program) y una dinámica de grupo extraída del libro «Mindfulness: una guía práctica para encontrar la paz en un mundo frenético» (Williams \& Penman, 2011/2013).

\section{Discusión}

El objetivo principal fue analizar la literatura científica sobre los efectos que tiene el entrenamiento en mindfulness en estudiantes universitarios. En este sentido, se han encontrado artículos que analizan los efectos a nivel psicológico y emocional (estrés, ansiedad, depresión, resolución de conflictos, resiliencia, bienestar, motivación), a nivel de rendimiento académico y dos estudios a nivel fisiológico (inmunología y parámetros cardiovasculares). A nivel general se han encontrado resultados positivos en todos los estudios a excepción de dos que no pudieron concluir mejorías significativas asociadas al entrenamiento en atención plena (Turner, et al., 2020; Wimmer, Bellingrath \& Von Stockhausen; 2020).

Respecto al propósito de analizar cuáles de estos estudios se aplican en la Educación Física Universitaria, no se han encontrado artículos que traten de manera específica con el mindfulness en esta área de conocimiento. Entendiendo los grandes beneficios que las prácticas de atención plena tienen sobre los deportistas tanto a nivel emocional como de rendimiento (Gooding \& Gardner, 2009; Kauffman, et al., 2009; Palmi \& Solé, 2016), se identifica la falta de estudios sobre mindfulness en la Educación Física Universitaria como un vacío de conocimiento científico a cubrir por futuros estudios.

En los siguientes subapartados se abordará el análisis y comparación en profundidad de las características y contenidos de cada uno de los 11 artículos incluidos en la pesquisa.

\section{País e idioma}

En primer lugar, cabe destacar una clara predominancia de artículos publi- 
cados en el Reino Unido, no existiendo ningún estudio que haya sido llevado a cabo en España. Esto es debido a la reciente implementación de estas técnicas de meditación en nuestro país (Mañas, 2009), no habiendo llegado aún a publicarse literatura científica sobre el ámbito
Universitario. Tampoco se han encontrado artículos publicados en Estados Unidos, lo cual es algo sorprendente por tratarse de uno de los países punteros en la aplicación del mindfulness en diferentes ámbitos (Clarke, et al., 2015).

Tabla 2

Resumen de los artículos de mindfulness publicados entre el 2014 y el 2020

\begin{tabular}{|c|c|c|c|c|c|}
\hline & Autor (año) & $\begin{array}{c}\text { País } \\
\text { (Idioma) }\end{array}$ & Sujetos (Duración) & $\begin{array}{l}\text { Tipo de } \\
\text { estudio }\end{array}$ & $\begin{array}{c}\text { Programa } \\
\text { mindfulness }\end{array}$ \\
\hline 1 & $\begin{array}{l}\text { Stevenson, } \\
\text { Millings, \& } \\
\text { Emerson } \\
(2019)\end{array}$ & $\begin{array}{l}\text { Inglaterra } \\
\text { (Inglés) }\end{array}$ & $\begin{array}{l}174 \text { estudiantes universitarios } \\
\text { (transversal) }\end{array}$ & $\begin{array}{l}\text { Cuantitativo: } \\
\text { encuesta en línea }\end{array}$ & Estudio descriptivo \\
\hline 2 & $\begin{array}{l}\text { Cavanagh et al. } \\
\text { (2018) }\end{array}$ & $\begin{array}{l}\text { Reino } \\
\text { Unido } \\
\text { (Inglés) }\end{array}$ & $\begin{array}{l}155 \text { estudiantes universitarios } \\
-2 \text { semanas } \\
-14 \text { sesiones desde casa } \\
(10 \mathrm{~min} / \text { sesión })\end{array}$ & $\begin{array}{l}\text { Cuantitativo: } \\
\text { cuestionarios }\end{array}$ & MBI y MBCT online \\
\hline
\end{tabular}

\begin{tabular}{|c|c|c|c|c|}
\hline Gu, Cavanagh, & Reino & 214 estudiantes universitarios & Cuantitativo: & MBSH online \\
\hline $\begin{array}{l}\text { \& Strauss } \\
\text { (2018) }\end{array}$ & $\begin{array}{l}\text { Unido } \\
\text { (Inglés) }\end{array}$ & $\begin{array}{l}-2 \text { semanas } \\
-14 \text { sesiones desde casa } \\
(10 \mathrm{~min} / \mathrm{sesión})\end{array}$ & cuestionarios & \\
\hline
\end{tabular}

Propósito Resultados

Examinar relación entre apego adulto, Los análisis de mediación revelaron que el afrontamiento atención plena disposicional y regulación del derrotismo fue mediador entre funcionamiento de emociones. mental resiliente y 5 escalas de bienestar psicológico y Investigar si estos constructos pueden entre funcionamiento emocional desorganizado y las 6 predecir el afrontamiento y el bienestar. escalas.

Comparar los efectos de dos $\quad$ MBI se asoció con mejoras significativas en los dominios intervenciones breves basadas en la medidos, con tamaños de efecto de pequeños a atención plena en línea (MBI; con y sin medianos. No hubo cambios significativos en grupo práctica de meditación formal) y un control.

grupo control sin intervención en una El cambio en pensamiento perseverativo media la muestra no clínica.

relación entre condición y mejora en estrés percibido y resultados de síntomas de ansiedad/depresión.

Examinar efectos de una intervención de Después de la intervención, se encontró que MBSH autoayuda basada en atención plena reduce significativamente el estrés en comparación con (MBSH) en línea comparado a dos ambas condiciones de control.

condiciones control: música clásica Los cambios en la atención plena, la autocompasión y la adaptada y lista espera sobre estrés percibido. preocupación median significativamente los efectos de Evaluar atención plena, autocompasión y sobre los cambios en el estrés. preocupación como mecanismos de los efectos de MBSH frente a ambas condiciones de control sobre el estrés.

\begin{tabular}{llllcc}
\hline 4 & James \& & Inglaterra & 60 estudiantes universitarios & Cuantitativo: & MBCT en casa con \\
Rimes (2018) & (Inglés) & -8 semanas & cuestionarios & grabaciones de \\
& & & \\
& & & \\
& & & \\
& & & \\
& & & \\
& & & \\
& & & \\
& & &
\end{tabular}

arar la terapia cognitiva basada en la Superioridad significativa de MBCT para los cambios en tención plena (MBCT) con una guía de las creencias inútiles sobre emociones, rumia, atención autoayuda basada en la terapia cognitivo- plena, autocompasión y descentramiento. Los efectos se conductual (CBT) para estudiantes mantienen en MBCT tras intervención. universitarios que experimentan Resultados superiores de MBCT para perfeccionismo y dificultades debido al perfeccionismo. deterioro diario causado por perfeccionismo. Mayor frecuencia de práctica se asoció con mayores mejoras en autocompasión.

\begin{tabular}{|c|c|c|c|c|c|c|c|}
\hline 5 & $\begin{array}{l}\text { Brzozowski, } \\
\text { Gillespie, } \\
\text { Dizon, \& } \\
\text { Mitchell } \\
\text { (2018) }\end{array}$ & $\begin{array}{l}\text { Inglaterra } \\
\text { (Inglés) }\end{array}$ & $\begin{array}{l}49 \text { estudiantes universitarios } \\
\text { (transversal) }\end{array}$ & $\begin{array}{l}\text { Cuantitativo: } \\
\text { cuestionarios }\end{array}$ & Estudio descriptivo & $\begin{array}{l}\text { Ampliar conocimiento sobre los } \\
\text { mecanismos del mindfulness en procesos } \\
\text { cardiovasculares. } \\
\text { Investigar la relación de la atención plena } \\
\text { con las emociones autoreportadas } \\
\text { durante un videoclip violento y las } \\
\text { respuestas cardiovasculares al clip. }\end{array}$ & $\begin{array}{l}\text { Sugieren que la atención plena está relacionada con la } \\
\text { reducción de los sentimientos negativos provocados por } \\
\text { los estímulos de movimiento violento. }\end{array}$ \\
\hline 6 & $\begin{array}{l}\text { Solhaug et al. } \\
\text { (2016) }\end{array}$ & $\begin{array}{l}\text { Noruega } \\
\text { (Inglés) }\end{array}$ & $\begin{array}{l}22 \text { estudiantes universitarios de } \\
\text { psicología y medicina } \\
-7 \text { semanas } \\
-7 \text { sesiones presenciales } \\
\text { (1.5 h/sesión) } \\
-49 \text { sesiones desde casa } \\
\text { (de } 20 \text { a } 45 \mathrm{~min} / \text { sesión) }\end{array}$ & $\begin{array}{l}\text { Cualitativo: } \\
\text { entrevistas a } \\
\text { grupos focales y } \\
\text { diez entrevistas } \\
\text { en profundidad }\end{array}$ & $\begin{array}{l}\text { MBSR combinado } \\
\text { presencial y en casa }\end{array}$ & $\begin{array}{l}\text { Explorar cómo los estudiantes de primer } \\
\text { año de medicina y psicología } \\
\text { experimentaron y conceptualizaron la } \\
\text { atención plena al completar un programa } \\
\text { de reducción del estrés basado en el } \\
\text { mindfulness de } 7 \text { semanas. }\end{array}$ & $\begin{array}{l}\text { Mayor atención y conciencia de fenómenos psicológicos } \\
\text { y corporales. La mayoría informó un cambio de actitud } \\
\text { hacia sus experiencias en términos de disminución de } \\
\text { reactividad, aumento de curiosidad, tolerancia afectiva, } \\
\text { paciencia, autoaceptación, y mejores cualidades } \\
\text { relacionales. }\end{array}$ \\
\hline 7 & $\begin{array}{l}\text { Senker, Fries, } \\
\& \text { Grund } \\
(2020)\end{array}$ & $\begin{array}{l}\text { Alemania } \\
\text { (Inglés) }\end{array}$ & $\begin{array}{l}56 \text { estudiantes universitarios } \\
-8 \text { días }\end{array}$ & $\begin{array}{l}\text { Cuantitativo: } \\
\text { cuestionarios }\end{array}$ & Estudio descriptivo & $\begin{array}{l}\text { Utilizar atención plena para mejorar } \\
\text { comprensión de los efectos de las } \\
\text { diferencias entre las personas cuando se } \\
\text { enfrentan situaciones de conflicto } \\
\text { motivacional. }\end{array}$ & $\begin{array}{l}\text { No juzgar (como un rasgo) y tener presencia } \\
\text { momentánea (como un estado), se relacionan con menor } \\
\text { intensidad en conflictos entre personas. Sugieren que ser } \\
\text { consciente es una tarea esencial de autorregulación, } \\
\text { beneficiosa para lidiar con conflictos motivacionales. }\end{array}$ \\
\hline 8 & $\begin{array}{l}\text { Wimmer, } \\
\text { Bellingrath, \& } \\
\text { von } \\
\text { Stockhausen } \\
(2020)\end{array}$ & $\begin{array}{l}\text { Alemania } \\
\text { (Inglés) }\end{array}$ & $\begin{array}{l}180 \text { estudiantes universitarios de } \\
\text { psicologia } \\
-12 \text { semanas } \\
-7 \text { sesiones presenciales } \\
(1.5 \mathrm{~h} / \text { sesión }) \\
-60 \text { sesiones desde casa } \\
(20 \mathrm{~min} / \text { sesión) }\end{array}$ & $\begin{array}{l}\text { Cuantitativo: } \\
\text { cuestionarios }\end{array}$ & MBI presencial & $\begin{array}{l}\text { Examinar efectos de entrenamiento en } \\
\text { mindfulness en la regulación de atención } \\
\text { y si los beneficios están influenciados por } \\
\text { el componente de yoga de las } \\
\text { intervenciones basadas en mindfulness } \\
\text { (MBI) y/o por la práctica de MBI. }\end{array}$ & $\begin{array}{l}\text { Con excepción de la velocidad en una tarea de } \\
\text { flexibilidad cognitiva, los resultados no indicaron ventaja } \\
\text { sistemática o diferencial derivada del entrenamiento de } \\
\text { la atención plena, con o sin yoga, con respecto a la } \\
\text { regulación de atención. No hubo influencia de la } \\
\text { cantidad o calidad de las tareas. }\end{array}$ \\
\hline 9 & $\begin{array}{l}\text { Moore et al. } \\
(2020)\end{array}$ & $\begin{array}{l}\text { Australia } \\
\text { (Inglés) }\end{array}$ & $\begin{array}{l}47 \text { estudiantes universitarios de } \\
\text { medicina } \\
-8 \text { semanas } \\
-40 \text { sesiones desde casa } \\
\text { (de } 5 \text { a } 10 \mathrm{~min} / \mathrm{sesión} \text { ) }\end{array}$ & $\begin{array}{l}\text { Cuantitativo: } \\
\text { cuestionarios y } \\
\text { cualitativo: } \\
\text { ensayo reflexivo }\end{array}$ & MTP online & $\begin{array}{l}\text { Determinar viabilidad y eficacia de un } \\
\text { programa de formación de mindfulness, } \\
\text { impartido } \\
\text { en línea a estudiantes de medicina en una } \\
\text { Escuela Clínica Rural. }\end{array}$ & $\begin{array}{l}\text { Reducción significativa en estrés percibido y aumento } \\
\text { significativo de autocompasión a los } 4 \text { meses de } \\
\text { seguimiento post-intervención. } \\
\text { Mejora de conocimientos sobre el impacto personal y } \\
\text { profesional de la meditación de atención plena, así como } \\
\text { de las barreras para la práctica. }\end{array}$ \\
\hline 10 & $\begin{array}{l}\text { Alzahrani et al. } \\
\text { (2020) }\end{array}$ & $\begin{array}{l}\text { Arabia Saudí } \\
\text { (Inglés) }\end{array}$ & $\begin{array}{l}289 \text { estudiantes universitarios de } \\
\text { medicina (transversal) }\end{array}$ & $\begin{array}{l}\text { Cuantitativo: } \\
\text { cuestionarios }\end{array}$ & Estudio descriptivo & $\begin{array}{l}\text { Examinar relación entre atención plena, } \\
\text { estrés, depresión y rendimiento } \\
\text { académico. } \\
\text { Explorar los posibles efectos de } \\
\text { moderación de la atención plena sobre el } \\
\text { impacto del estrés en el rendimiento } \\
\text { académico y la depresión. }\end{array}$ & $\begin{array}{l}\text { La atención plena se asocia inversamente con depresión } \\
\text { y estrés, pero no con rendimiento académico. } \\
\text { La regresión logistica múltiple mostró que la atención } \\
\text { plena puede predecir tanto depresión como estrés. } \\
\text { Dos subescalas de atención plena pueden moderar la } \\
\text { relación entre estrés y depresión: no juzgar la } \\
\text { experiencia interior y describir. }\end{array}$ \\
\hline 11 & $\begin{array}{l}\text { Turner et al. } \\
\text { (2020) }\end{array}$ & $\begin{array}{l}\text { Inglaterra } \\
\text { (Inglés) }\end{array}$ & $\begin{array}{l}27 \text { estudiantes universitarios } \\
-8 \text { semanas } \\
-8 \text { sesiones presenciales } \\
\text { (de } 75 \text { a } 90 \mathrm{~min} / \text { sesión) }\end{array}$ & $\begin{array}{l}\text { Cuantitativo: } \\
\text { cuestionarios }\end{array}$ & $\begin{array}{l}\text { Habilidades grupales } \\
\text { a partir de un } \\
\text { programa del libro: } \\
\text { Mindfulness: Guía } \\
\text { práctica para encontrar } \\
\text { la paz en un mundo } \\
\quad \text { frenético. }\end{array}$ & $\begin{array}{l}\text { Estudiar la desregulación inmunológica } \\
\text { en una cohorte de estudiantes expuestos a } \\
\text { un estrés progresivamente mayor hacia el } \\
\text { período de exámenes, y explorar si el } \\
\text { entrenamiento de atención plena mitigó } \\
\text { esta desregulación. }\end{array}$ & $\begin{array}{l}\text { El aumento de la angustia se asoció con aumentos } \\
\text { significativos en la proporción de células B, } \\
\text { independientemente del grupo de prueba. } \\
\text { No encontraron evidencias de que el entrenamiento de } \\
\text { atención plena pueda amortiguar los efectos de la } \\
\text { angustia psicológica en el sistema inmunológico. }\end{array}$ \\
\hline
\end{tabular}

Notas. min: minutos; h: horas; MBI: intervenciones basadas en mindfulness (del inglés mindfulness-based interventions); MBCT: terapia cognitiva basada en mindfulness (del inglés mindfulness-based cognitive therapy); MBSR: reducción del estrés a través del mindfulness (del inglés mindfulness-based stress reduction); MBSH: autoayuda basada en mindfulness (del inglés mindfulness-based self help); MTP: programa de entrenamiento en mindfulness (del inglés mindfulness training program). 


\section{Muestra y duración del estudio}

Comprendiendo que los adultos jóvenes están desarrollando su identidad y se caracterizan por cierta inestabilidad (Cavanagh, et al., 2018; Senker, Fries \& Grund, 2020), se hace necesaria la implementación de este tipo de técnicas de atención plena en esta población. Los tamaños muestrales empleados varían ampliamente de un estudio a otro, aunque numerosos estudios se respaldan en un análisis de potencia previo. Esta varianza entre el número de sujetos es debida principalmente a los diseños de estudio. Mientras que procedimientos arduos como toma de muestras sanguíneas (Turner, et al., 2020) dificultan el reclutamiento de participantes, las autointervenciones con vídeos o similares a distancia o los procedimientos mixtos (presencial y a distancia) facilitan el proceso de investigación y la adherencia (Solhaug, et al., 2016; Wimmer, et al., 2020). Además de lo anterior, las características de los participantes en términos de cultura, rama de estudios y otros, podrían influir en los resultados de las investigaciones (Stevenson, Millings \& Emerson, 2019). En este sentido, mientras que la mayoría de los estudios sólo indican datos demográficos de la muestra como sexo o edad, sólo cuatro artículos seleccionan a los estudiantes en función de su rama de conocimiento; dos artículos incluyendo estudiantes de medicina (Alzahrani, et al., 2020; Moore, et al., 2020), uno de psicología (Wimmer, et al., 2020), y uno incluyendo ambas ramas de estudio (Solhaug, et al., 2016). También son pocos los estudios que indican la experiencia previa de los participantes en prácticas de mindfulness, lo cuál podría condicionar los efectos interpersonales (Brzozowski, Gillespie, Dizon, \& Mitchell, 2018).

Respecto a la duración de las intervenciones, cabe destacar en primer lugar que hay cuatro estudios exclusivamente descriptivos que no entrenan a los participantes, si no que miden la capacidad de atención plena de que disponen los sujetos para relacionarla con otros parámetros (Alzahrani, et al., 2020; Brzozowski, et al., 2018; Senker, et al., 2020; Stevenson, et al., 2019). Dentro de estos estudios descriptivos, son todos transversales (con mediciones un sólo día) excepto el de Senker et al. (2020), que toma datos de los participantes durante 8 días consecutivos. Los otros siete estudios varían entre dos y 12 semanas de intervención. En este sentido, algunos autores aseguran que para llegar a asimilar los aspectos positivos del mindfulness es necesario, como mínimo, un proceso de ocho semanas (Kiens \& Larsen, 2020; Yook, Kang \& Park, 2017). Cabe destacar igualmente el artículo de Moore et al. (2020), en el que, tras ocho semanas de intervención, se vuelven a tomar medidas cuantitativas y cualitativas cuatro meses después para evaluar la evolución en el tiempo de la intervención. Dentro de estos tiempos de intervención, el número de sesiones empleadas y la duración de cada sesión difieren aún en mayor medida (ver tabla 2). Aunque se ha demostrado que versiones cortas de programas de mindfulness pueden tener los mismos efectos beneficiosos (Solhaug, et al., 2016), se sobreentienden resultados muy dispares entre que las sesiones duren cinco minutos o dos horas.

\section{Tipo de investigación}

Se ha podido observar que la gran mayoría de las investigaciones (cinco de las 7) utilizan una intervención online o mixta. Este hecho podría conllevar un sesgo a la hora de comparar los resultados entre los diferentes estudios y extraer conclusiones (Miró, et al., 2011).

Respecto a la metodología utilizada en la recogida de datos, literatura científica previa ha demostrado que la recogida de datos auto-reportados es comparable tanto si se realiza a través del método «lápiz-papel» como a través de internet (Cavanagah, et al., 2018; Wimmer, et al., 2020). En este sentido, gran parte de los estudios utilizan metodologías cuantitativas a través de cuestionarios (diez de los 11 totales) y sólo dos de ellos entrevistas (Moore, et al., 2020; Solhaug, et al., 2016). Cabe destacar la desigualdad en el número de artículos que recogen datos cualitativos para la evaluación de la efectividad de las metodologías analizadas, lo que reafirma los hallazgos de otros autores (Atkinson, Coffey \& Delamont, 2003; Campayo \& Alarcón, 2017). En líneas de intervenciones enfocadas al bienestar psicológico y emocional de los estudiantes como lo es el mindfulness, los datos cuantitativos son de gran relevancia para el análisis de parámetros de bienestar, autoeficiencia y autorregulación, entre otros. Sin embargo, para estas investigaciones con numerosas variables de carácter abstracto, sería de vital importancia incluir análisis cualitativos que permitan profundizar en las percepciones subjetivas de los participantes sobre los beneficios, ventajas y desventajas de los programas de mindfulness aplicados.

\section{Propósito, resultados y programa mindfulness utilizado}

La atención plena es una capacidad natural que puede ser potenciada mediante entrenamiento (Senker, et al., 2020). Numerosos programas de entrenamiento del 
mindfulness con características propias han sido diseñados en los últimos años. Concretamente, se utilizó la intervención basada en mindfulness (MBI) y la terapia cognitiva basada en mindfulness (MBCT) en dos estudios diferentes cada programa; la reducción del estrés a través del mindfulness (MBSR), la autoayuda basada en mindfulness (MBSH) y el programa de entrenamiento en mindfulness (MTP) fueron utilizadas en una investigación cada una. Además, se utilizó en un estudio una dinámica grupal extraída del libro deWilliams y Penman (2011/2013) como intervención.

Respecto a los objetivos de las investigaciones incluidas se observa un enfoque predominante hacia parámetros de salud psicológica (estrés, ansiedad, depresión, motivación, autoaceptación) con buenos resultados en la mayoría de los estudios (Alzahrani, et al., 2020; Cavanagah, et al., 2018; Gu, Cavanagh \& Strauss, 2018; James \& Rimes, 2018; Moore, et al., 2020; Senker, et al., 2020; Solhaug, et al., 2016; Stevenson, et al., 2019) y sólo un estudio obteniendo resultados no significativos tras un MBI combinado con yoga (Wimmer, et al., 2020). Además, dos estudios analizaron variables fisiológicas (cardiovasculares e inmunológicas). En estos, se observó una relación entre el dominio del mindfulness y una frecuencia cardíaca basal elevada, lo cual puede ser un factor de riesgo coronario (Brzozowski, et al., 2018). Al mismo tiempo, mientras que no se pudo asociar el mindfulness a parámetros inmunológicos, el aumento de la angustia sí que apareció asociado a una desregulación inmunológica con procesos proinflamatorios (Turner, et al., 2020). Respecto al uso específico de los programas, se demuestra que las intervenciones basadas en mindfulness (MBI) optimizan dominios de ansiedad, depresión y estrés (Cavanagah, et al., 2018; Wimmer, et al., 2020); la autoayuda basada en mindfulness (MBSH) y el programa de entrenamiento en mindfulness (MTP) reducen significativamente el estrés (Gu, et al., 2018; Moore, et al., 2020); la terapia cognitiva basada en atención plena (MBCT) mejora las creencias sobre emociones, autocompasión, perfeccionismo y descentramiento (James \& Rimes, 2018); y por último, la reducción del estrés a través del mindfulness (MBSR) conlleva un progreso en términos de disminución de reactividad, aumento de curiosidad, tolerancia afectiva, paciencia, autoaceptación, y cualidades relacionales (Solhaug, et al., 2016). A parte de los programas concretos, los estudios descriptivos muestran correlaciones significativas entre la capacidad de atención plena y los siguientes parámetros de bienestar psicológico: a) la depresión y el estrés (correlación ne- gativa; Alzahrani, et al., 2020); b) la autorregulación en conflictos motivacionales (correlación positiva; Senker, et al., 2020); c) los sentimientos negativos (correlación negativa; Brzozowski, et al., 2018); y d) diferentes parámetros de bienestar psicológico (Stevenson, et al., 2019). No se pudo relacionar la capacidad de atención plena con el rendimiento académico (Alzahrani, et al., 2020).

\section{Limitaciones y futuras líneas de investiga- ción}

En primer lugar, cabe remarcar la variabilidad (programa aplicado, duración) entre los programas de mindfulness que han sido utilizados en cada estudio. Esto dificulta la capacidad de extraer conclusiones concretas y más bien aboca a generalizaciones. Se ha observado una limitación en términos de intervenciones enfocadas a la Educación Física Universitaria. Futuros estudios deberían abordar este ámbito y más aún, teniendo en cuenta los beneficios que tiene el mindfulness a nivel deportivo sumados a los que se han visto en la presente investigación. En segundo lugar, una limitación es el periodo seleccionado. Se propone, para futuras líneas de investigación, ampliar el periodo temporal en diez años y realizar una comparativa con los resultados obtenidos en este estudio. Además, se propone revisar los criterios de inclusión e incluir revistas no indexadas en JCR. Por último, sería interesante comprobar los efectos de estos programas en profesores para de esta manera, tratar de obtener mejorías en la calidad de la enseñanza.

\section{Conclusiones}

Esta investigación aporta un análisis bibliográfico conciso y objetivo de los estudios científicos publicados sobre atención plena (mindfulness) en contextos educativos universitarios. Este tipo de investigación representa una novedad, ya que no existen revisiones sistemáticas en los últimos seis años sobre los efectos del mindfulness en sujetos universitarios. En los estudios incluidos se evalúan tanto programas de entrenamiento de mindfulness y sus efectos, como relaciones entre capacidad de atención plena y diferentes aspectos de bienestar psicológico. A nivel general y manteniendo el objetivo principal en mente, se ha podido constatar que el entrenamiento de la atención plena conlleva beneficios en los estudiantes universitarios a nivel psicológico, cognitivo y emocional, entre otros. Respecto a las adaptaciones fisiológicas, habría que ser cautos a la hora de 
extraer conclusiones, pues sólo han sido analizadas por dos estudios y sin hallazgos significativos. Cabe destacar la falta de estudios en el área de la Educación Física Universitaria. Además, se ha identificado la necesidad de realizar intervenciones de mindfulness en Universidades españolas para certificar su validez en estudiantes con dichas características socioculturales. Todas las conclusiones presentadas en este trabajo deben ser tomadas con cautela, dado que se han analizado un número limitado de estudios en un periodo de tiempo concreto y los mismos han utilizado programas variados, así como preguntas y metodologías de investigación diversas.

\section{Referencias}

Alzahrani,A.M.,Hakami,A.,AlHadi,A., Batais,M.A.,Alrasheed,A.A., \& Almigbal,T.H. (2020). The interplay between mindfulness, depression, stressandacademicperformance inmedical students: A Saudiperspective. PlosOne, 15(4), 1-11.https://doi.org/10.1371/ journal.pone.0231088

Anand, U., \& Sharma, M. P. (2014). Effectiveness of a mindfulnessbased stressreduction program on stress and well-beinginadolescents in a school setting. Indian Journal of Positive Psychology, 5(1), 17.

Aránega, A.Y., Sánchez, R. C., \& Pérez, C. G. (2019). Mindfulness' effects on undergraduates' perception of self-knowledge and stress levels. Journal of Business Research, 101,441-446.

Atkinson, P., Coffey,A., \& Delamont, S. (2003). Key themes in qualitative research.Walnut Creek, CA, USA:Altamira.

Baer R.A., Lykins E.L.B., \& Peters J.R. (2012). Mindfulness and selfcompassion as predictors of psychological well-being in long-term meditators and matched non-meditators. Journal of Positive Psychology, 7, 230-238.

Bandura,A.(2005).The primacy of self-regulation in healthpromotion. Applied Psychology, 54(2), 245-254.

Barba-Martín, R.A., Bores-García,D., Hortigüela-Alcalá,D.,\& GonzálezCalvo, G. (2020). The application of the teaching games for understanding in physical education. Systematic review of the last six years. International Journal of Environmental Research and Public Health, 17(9), 3330.

Birrer,D., Röthlin, P., \& Morgan, G. (2012). Mindfulness to enhance athleticperformance:Theoretical considerationsand possibleimpact mechanisms. Mindfulness, 3, 325-246.

Brzozowski,A., Gillespie, S.M., Dixon, L., \& Mitchell, I.J. (2018). Mindfulness dampens cardiacresponses tomotionscenes of violence. Mindfulness, 9(2), 575-584.

Campayo, J., \& Alarcón, M. (2017). Evidencias científicas de mindfulness aplicadas a la educación. Desarrollando la atención plena en las aulas [ponencia]. En J. Campayo (Presidencia), International Meetingon Mindfulness, Zaragoza, España.

Carraça, B., Serpa, S., Rosado,A., \& Guerrero, P. (2019). Mindfulness and Compassion Strategies on Elite Soccer-Conceptualization of Mindfulness-Based Soccer Program(MBSoccerP). Biomedical Journal of Scientific \&Technical Research, $14(2), 10565-10571$.

Carraça, B., Serpa, S., Rosado,A., \& Palmi, J. (2018).The Mindfulness-
Based Soccer Program (MBSoccerP): Effects on Elite Athletes. Cuadernos de Psicología del Deporte, 18(3), 62-85.

Cavanagh, K., Churchard,A., O’Hanlon, P., Mundy,T.,Votolato, P., Jones,F., Gu,J., \& Strauss, C. (2018).A randomised controlled trial of a brief online mindfulness-based intervention in a non-clinical population: replicationand extension. Mindfulness, 9(4), 1191-1205.

Clarke,T.C., Black, L.I., Stussman, B.J., Barnes, P.M., \& Nahin, R.L. (2015).Trends in the use of complementary health approaches among adults: United States, 2002-2012. National Health Statistics Reports, 79, 1-16.

Cuéllar,R., Bazán,A., \&Araya, G. (2018). Efectos delapráctica deaikido sobre mindfulness y la ansiedad en estudiantes universitarios de Costa Rica (Effects of practicing aikido on mindfulness and anxiety inCostaRicanuniversitystudents). Retos, 35, 13-19.https://doi.org/ 10.47197/retos.v0i35.62044

Dunn, B.R.,Hartigan,J.A., \& Mikulas,W.L.(1999). Concentrationand mindfulness meditations: unique forms of consciousness?Applied psychophysiologyandbiofeedback, 24(3), 147-165.

Ergas, O., \& Hadar,L.L. (2019). Mindfulnessinandas education:A map of a developing academic discourse from 2002 to 2017. Review of Education, 7(3), 757-797.

Fortney L., Luchterhand C., \& Zakletskaia L. (2013). Abbreviated mindfulness intervention for job satisfaction, quality of life, and compassion in primary care clinicians: apilot study. Annals of Family Medicine, 11,412-420.

Gallego, J.,Aguilar-Parra,J., Cangas,A.,Langer, Á, \& Mañas,I.(2014). Effect of a Mindfulness Program on Stress,Anxiety andDepression in University Students. The Spanish Journal of Psychology, 17, E109. doi:10.1017/sjp.2014.102

Garcés,E.J.,DeFrancisco, C., \&Arce, C.(2012). Inventario de Burnout en Deportistas Revisado (IBD-R). Revista de Psicología del Deporte, 21(2), 271-278.

Gardner,F.L. (2009). Efficacy, mechanisms of change, and the scientific development of sport psychology. Journal of Clinical Sport Psychology, 3,139-155.

Gooding,A., \& Gardner,F.L.(2009).Aninvestigation of the relationship between mindfulness, preshot routine, and basketball free throw percentage. Journal of Clinical Sport Psychology, 3(4), 303-319.

Greeson, J.M., Juberg, M.K., Maytan, M., James, K., \& Rogers, H. (2014). A randomized controlled trial of Koru: A mindfulness program for college students and other emerging adults. Journal of American College Health, 62(4), 222-233.

GrossmanP.,NiemannL.,SchmidtS., \&WalachH.(2004). Mindfulnessbased stress reduction and health benefits:A meta-analysis. Journal of Psychosomatic Research, 57, 35-43.

Gu, J., Cavanagh, K., \& Strauss, C. (2018). Investigating the specific effects of an online mindfulness-based self-help intervention on stress and underlyingmechanisms. Mindfulness, 9(4), 1245-1257.

Hart, R., Ivtzan, I., \& Hart, D. (2013). Mind the gap in mindfulness research:A comparative account of the leading schools of thought. Review of General Psychology, 17(4),453-466.

Hernández-Amorós, M.J., \& Urrea-Solano, M.E. (2019).Evaluación deun taller demindfulness: laapuestapor una tutoría máshumanista en la Facultad deEducación. En R. Roig-Vila(Ed.), Investigacióne innovación en la Enseñanza Superior: Nuevos contextos,nuevas ideas (pp. 
956-966). Octaedro.

Hülsheger U.R., Lang J.W.B., Depenbrock F., Fehrmann C., Zijlstra F.R.H., \&Alberts H.J.E.M. (2014).The power of presence:The role of mindfulness at work for daily levels and change trajectories of psychological detachment and sleep quality. Journal of Applied Psychology, 99, 1113-1128.

James, K., \& Rimes, K.A. (2018). Mindfulness-based cognitive therapy versus pure cognitive behavioural self-help for perfectionism: a pilotrandomised study. Mindfulness, 9(3), 801-814.

Kabat-Zinn,J.(1994). Wherever you go, there you are:Mindfulness meditation in everyday life. NW: Hyperio.

Kabat-Zinn, J.(2003). Mindfulness based intervention in context:past, present and future. Clinical Psychology: Science and Practice, 10(2), 144-146.

Kauffman, K.A., Glass, C.R., \& Arnkoff, D.B. (2009). Evaluation of Mindful SportPerformanceEnhancement(MSPE):Anewapproach to promote flow in athletes. Journal of Clinical Sports Psychology, 4, 334-356.

Kaufman, P. (2017). Critical contemplative pedagogy. Radical Pedagogy, 14, 15246345.

Kiens,K.,\&Larsen,C.H.(2020). Provision of amental skillsintervention program in an elite sport school for student-athletes. Journal of Sport Psychology inAction, 1-15.

Mahalingam, R., \& Rabelo, V.C. (2019). Teaching mindfulness to undergraduates: a survey and photovoice study. Journal of Transformative Education, 17(1), 51-70.

Mañas, I. (2009). Mindfulness (AtenciónPlena): Lameditación en psicología clínica. Gaceta de Psicología, 50, 13-29.

Martínez-Rubio, D., Sanabria-Mazo, J.P., Feliu-Soler,A., ColomerCarbonell,A., Martínez-Brotóns, C., Solé,S., ...\& Montero-Marín, J. (2020). Testing the intermediary role of perceived stress in the relationship between mindfulness and burnout subtypes in a large sample of Spanish university students. International Journal of Environmental Research and Public Health, 17(19), 7013. https: / / doi.org/10.3390/ijerph17197013

Minkler,T.O., Glass, C.R., \& Hut, M.(2020). Mindfulness trainingfor a college team: Feasibility, acceptability, and effectiveness from within an athletic department. Journal of Applied Sport Psychology, 118.

Miró, M.T., Perestelo-Pérez,L., Pérez,J., Rivero,A., González, M., Fuente,J.,\& Serrano,P.(2011).Eficaciadelos tratamientosbasados en mindfulness para los trastornos de ansiedad y depresión: una revisión sistemática. Revista de Psicopatologíay Psicología Clínica, 16(1), $1-14$.

Moore, S., Barbour, R., Ngo,H., Sinclair, C., Chambers, R.,Auret,K., ... \& Playford, D. (2020). Determining the feasibility and effectiveness of brief online mindfulness training for rural medical students: apilotstudy. BMCMedical Education, 20,1-12.

Mrazek, M.D., Franklin, M.S., Phillips,D.T., Baird, B. \& \& Schooler,J.W. (2013). Mindfulness training improves workingmemory capacity and GREperformance whilereducingmind wandering. Psychological science, 24(5), 776-781.

Palmi, J., \& Solé, S. (2016). Intervenciones basadas en mindfulness (atención plena) en psicología del deporte. Revista de Psicología del Deporte, 25, 147-155.

Ryan, R.M., \& Deci, E.L. (2007). Active human nature: Self- determination theoryand the promotionandmaintenance of sport, exercise, and health. En M. S. Hagger y N. Chatzisarantis (Eds.), Intrinsic motivation and self-determination in exercise and sport (pp. 119). Champaign, IL: Human Kinetics.

Schneider,J., Malinowski, P.,Watson,P.M., \& Lattimore, P.(2019).The role of mindfulness in physical activity:A systematic review. Obesity Reviews, 20(3), 448463.

Senker, K., Fries, S., \& Grund,A. (2020). Mindfulness in everyday life: between-and within-person relationships to motivational conflicts. Current Psychology, 1-16.

Shapiro, S.L., \& Carlson, L.E. (2017). TheArt and Science of Mindfulness: Integrating Mindfulness into Psychology and the Helping Professions. Washington,DC:American Psychological Association.

Shapiro, S.L., Carlson, L.E., Astin, J.A., \& Freedman, B. (2006). Mechanisms of mindfulness. Journal of Clinical Psychology, 62(3), 373-386.

Solé, S., Carrança, B., Serpa, S., \& Palmi, J. (2014).Aplicaciones del mindfulness (concienciaplena) en lesión deportiva. Revista de Psicología del Deporte, 23, 501-508.

Solhaug, I., Eriksen,T.E., deVibe, M., Haavind, H., Friborg, O., Sørlie, T., \& Rosenvinge, J.H. (2016). Medical and psychology student's experiencesinlearningmindfulness: benefits, paradoxes, and pitfalls. Mindfulness, 7(4), 838-850.

Stevenson,J.C., Millings,A., \& Emerson,L.-M. (2019). Psychological well-being and coping: the predictive value of adult attachment, dispositional mindfulness, andemotionregulation. Mindfulness, 10(2), 256-271.

Trujillo-Torrealva, D., \& Reyes-Bossio, M. (2019). Programa de mindfulness para la reducción de la ansiedad precompetitiva en deportistas deartesmarciales. Retos, 36, 418-426. https: / /doi.org/ 10.47197/retos.v36i36.66589

Turner, L., Galante, J., Vainre, M., Stochl, J., Dufour, G., \& Jones, P.B. (2020). immune dysregulation among students exposed to exam stress and its mitigation by mindfulness training: findings from an exploratory randomised trial, Scientific Reports, 10(1), 1-11.

Urrútia, G., \& Bonfill, X. (2010). Declaración PRISMA: una propuesta paramejorar la publicación de revisiones sistemáticas ymetaanálisis. Medicina Clínica, 135(11), 507-511.

Whitmarsh, S., Uddén, J., Barendregt,H., \& Petersson, K.M. (2013). Mindfulness reduces habitual respond-ing based on implicit knowledge:Evidence fromartificial grammar learning. Consciousness and Cognition, 22(3), 833-845.

Williams, M., \& Penman, D. (2013). Mindfulness: Guía práctica para encontrar la paz en un mundo frenético (Remedios Diéguez, trad.). Barcelona: Paidós. (Obra original publicada en 2011).

Wimmer,L., Bellingrath,S., \&VonStockhausen,L.(2020). Mindfulness trainingfor improving attention regulation in university students: is it effective? and do yoga and homework matter? Frontiers in Psychology, 11(719), 1-15.

Worthen, D., \& Luiselli, J.K. (2016). Attitudes and opinions of female high school athletes about sports-focusedmindfulness training and practices. Journal of Clinical Sport Psychology, 10(3), 177-191.

Yook, Y.S., Kang, S.J., \& Park, I. (2017). Effects of physical activity intervention combining a new sport and mindfulness yoga on psychological characteristics in adolescents, International Journal of Sport and Exercise Psychology, 15(2), 109-117. 